\title{
Auditory Selective Attention: An fMRI Investigation
}

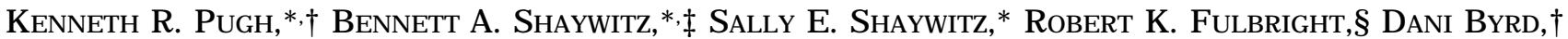 \\ Pawel Skudlarski,§ Donald P. Shankweiler, $\dagger+\|$ Leonard Katz, $\dagger \|$ R. Todd Constable,§ J ack Fletcher, ** \\ Cheryl Lacadie,§ Karen Marchione,* and J ohn C. Gore§'†† \\ *Department of Pediatrics, ¥Department of Neurology, and §Department of Diagnostic Radiology, YaleU niversity School of Medicine, \\ New Haven, Connecticut 06510; †Haskins Laboratories, New Haven, Connecticut; and ††Department of Applied Physics, YaleUniversity, \\ New Haven, Connecticut 06510; | Department of Psychology, University of Connecticut, Storrs, Connecticut 06268; and \\ **Department of Pediatrics, School of Medicine, University of Texas-Houston, Houston, Texas 77225
}

Received April 22, 1996

\begin{abstract}
In the present experiment, 25 adult subjects discriminated speech tokens ([ba]/[da]) or made pitch judgments on tone stimuli (rising/falling) under both binaural and dichotic listening conditions. We observed that when listeners performed tasks under the dichotic conditions, during which greater demands are made on auditory selective attention, activation within the posterior (parietal) attention system and at primary processing sites in the superior temporal and inferior frontal regions was increased. The cingulate gyrus within the anterior attention system was not influenced by this manipulation. Hemispheric differences between speech and nonspeech tasks were also observed, both at Broca's Area within the inferior frontal gyrus and in the middle temporal gyrus. 1996 Academic Press, Inc.
\end{abstract}

\section{THE STUDY OF ATTENTION WITHIN COGNITIVE PSYCHOLOGY}

Attention is a very broad term and arriving at a consensus regarding operational definitions of its various components has been one of the thorniest problems faced by cognitive scientists (All port, 1989; Kahneman, 1973; Posner and Boies, 1971; Schneider and Shiffrin, 1977; Wickens, 1980). When considering attention at the cognitive level, we must consider the following functional issues. First, an individual operating in an environment is bombarded by a vast array of perceptual inputs simultaneously and must, in order to function effectively, somehow select certain things for enhanced processing while ignoring others (Allport, 1989; Posner, 1991). (Sel ective attention may, of course, befurther subdivided into operations such as disengagement from a current focus, engagement of a new focus, and sustained focal attention over time (Posner and Peterson, 1990; Posner, 1991).) Second, there appear to belimits on the number of things that can be processed simultaneously; that is, a bottleneck or capacity limitation exists on the ability to divide attention between multiple stimuli or mental events.

Much early laboratory work in experimental psychology was focused on selective attention, using, for example, the dichotic listening paradigm. In this paradigm subjects receive, simultaneously, different messages to the left and right ear and must selectively attend to only one. I nitial studies suggested that information from the unattended channel was filtered out very early in processing (cf. Broadbent, 1958). If not instructed to switch attention to the unattended channel within approximately $2 \mathrm{~s}$ of stimulus onset the subject's ability to report the stimulus event in that channel was negligible. This led to a conceptualization of selective attention as analogous to a mechanical filter that could be adjusted to allow some messages to pass through while effectively blocking others at some precognitive sensory stage. However, later work (cf. Treisman, 1964) challenged this notion and demonstrated that information in the unattended channel was, in fact, processed to a cognitive-semantic level. Findings of this sort led to so-called late selection models in which information from all channels is thought to be perceived and processed to some extent, with selection operating at a more central locus, within the constraints of short-term/working memory. The fact that not all information can be processed to a point where the subject lays down some episodic memory for the event pointed to what appeared to be a bottleneck or capacity limitation at the level of higher mental processes.

Initial work aimed at understanding the nature of the apparent capacity limitations associated with divided attention often employed dual task paradigms (Posner and Boies, 1971). In this sort of paradigm, performance on a given cognitive task is measured under two conditions, alone and in conjunction with a second task. Decrements in task performance associ- 
ated with the simultaneous performance of the second task can be seen as a measure of the attentional demands made by that second task. I nitial research of this sort suggested a limited-capacity central attentional resource that can be allocated to different tasks but with corresponding performance costs as a function of the relative demands of each (see Kahneman, 1973). However, further work revealed a more complex state of affairs wherein dual task interference was obtained with some task combinations but not with others (Navon and Gopher, 1979). This, in turn, suggested to many researchers that the notion of a unitary and central attentional resource system as the key to capacity limitations was inadequate. The notion that the brain contains multiple attentional control systems, each of which might be associated with the control of somewhat different repertoires of behaviors, became more prevalent (Navon, 1984).

This multiple resource/system view appears to be most consistent with suggestions from neuropsychological research. Studies of patients with neurological damage, along with imaging experiments, suggest at minimum two attentional control systems associated with distinct anterior and posterior sites in the brain (Allport, 1989; Posner and Peterson, 1990; Posner and Dehaene, 1994). In one such conceptualization, the anterior system is thought to be associated with the control of decision and action and the posterior system with the control of perception (Posner and Dehaene, 1994). A brief review of the literature relevant to the neuropsychology of attention is provided in the following section.

\section{NEURO PSYCHOLOGICAL STUDIES OF ATTENTION}

A number of empirical studies and several theoretically oriented reviews have posited localized neural systems associated with aspects of attention such as sustained attention, control of selective attention, and control of decision and action (cf. Posner and Peterson, 1990; Posner and Dehaene, 1994). The relevant studies include both examinations of patients with identifiable lesions in different brain regions and recent studies using neuroimaging techniques.

\section{Sustained Attention}

Studies of sustained attention (vigilance) have implicated a greater role for right hemisphere sites than left hemisphere sites. For example, in a positron emission tomography (PET) study, Pardo and colleagues had subjects continuously monitor for subtle changes in both visual and somatosensory stimuli and found strong activation in right hemisphere $(\mathrm{RH})$ superior parietal sites and in RH prefrontal sites for both types of stimuli (Pardo et al., 1991). Lesions of the right hemisphere are often associated with deficits in responsiveness to warning signals intended to increase alertness (Heilman et al., 1985), and the maintenance of an alert state is obviously a critical component of good performance in vigilance tasks (Posner and Peterson, 1990).

\section{Selective Attention}

The control of selective attention, which requires that the organism target some sensory dimension for focal processing while effectively inhibiting others, should be associated with two related and measurable brain phenomena. First, cognitive systems that control the attentional shift and the engage/disengage operations at the core of selective attention should be active when task demands stress any or all of these component operations. Second, these controlling operations should produce consequent changes in the responsiveness and activation states of cortical and/or subcortical regions that process information relevant to a particular sensory dimension (Posner and Dehaene, 1994; Roland, 1994).

Posner (Posner and Presti, 1987, Posner and Petersen, 1990; Posner, 1987; Posner and Dehaene, 1994) has provided extensive reviews of neuropsychological studies of visual selective attention that suggest that the superior parietal lobule ( $\mathrm{RH}$ more than $\mathrm{LH}$ ) is most strongly associated with disengaging attention from its current focus to a new one, that the superior colliculus is most strongly associated with shifting to the new focus (engagement), and that the pulvinar is most strongly associated with filtering out irrel evant information from competing stimulation.

Beyond the visual domain, it is also the case that right hemisphere parietal lesions have been shown to produce problems with attending to tactile and auditory channels (Posner and Presti, 1987). I mportantly, deficits in different sensory modalities tend to be uncorrelated within the individual patient. This implies that different sites within the parietal lobearelikely responsible for control of selective attention in different modalities. The present study provides results rel evant to auditory selective attention by using both speech and nonspeech auditory perception tasks. Auditory selective attention has been, to date, less studied than visual selective attention. Woldorff et al. (1993) and Hari et al . (1989) both observed increased neuromagnetic field activation increases associated with selective attention in what appeared to be the supratemporal auditory cortex, but reported no changes at other posterior sites.

The effects of increased focal attention on primary and secondary sensory regions appear to be increased metabolism at these sites and decreased activation at others, even in theabsence of overt stimulation. Roland (1994) suggests that the effect of selective attention is to increase the EPSPs in these regions in anticipation 
of a stimulus event. Studies using electrophysiological measures indeed suggest that when stimuli are delivered to attended-to regions of the environment, responses are enhanced, but when delivered to unattended regions responses are suppressed (Posner and Dehaene, 1994). Whatever the precise mix of excitation and inhibition may be, the foregoing suggests that a physiological understanding of selective attention should be informed both by identification of modality relevant control sites and by examination of the relative activation and deactivation levels in the sensory networks associated with stimulus processing.

\section{Attention in Control of Decision and Action}

In contrast to systems that control perception, it is necessary to consider systems that control mental operations involved in decision making and action consequent on those decisions. These control processes seem to be most strongly associated with anterior brain systems (Posner and Dehaene, 1994).

The anterior cingulate gyrus appears to play a critical role in tasks that require complex cognitive processing. For example, Corbetta and colleagues (1991) found that in a task in which subjects monitored visual displays for several independent features in parallel (a divided attention task), cingulate activation was more pronounced relative to selective attention conditions. Similarly, finding a semantically relevant verb to describe the function of a common noun activates this region strongly (Petersen and Fiez, 1993); yet when the same stimuli are repeated many times, thereby allowing for memorized responses, this region is no longer active (Raichle et al., 1994). In the classic Stroop interference paradigm, in which a naming response is made in the presence of competing perceptual information, this region is strongly activated (Pardo et al., 1990). Working memory tasks, i.e., tasks that tap executive function heavily, have been shown to activate frontal areas extensively (cf. Petrides et al., 1993). While the various components of executive function have not been clearly localized, there is not much doubt that many complex cognitive operations are controlled by frontal lobe systems (Goldman-Rakic, 1987).

\section{Predictions}

The distinction between posterior attentional systems responsible for the control of perception versus anterior attentional systems responsible for the control of decision and action provide the framework for the specific hypotheses addressed by the present study. The work reported below used a set of auditory tasks performed under conditions that vary demands made on selective attention while holding constant the demands made on decision and action. In the most general sense, we anticipated that the current experi- ment would provide information regarding the operation and location of the posterior attention control sites most rel evant to the auditory modality. A $2 \times 2$ auditory discrimination paradigm was used. In one task, subjects judged whether a given synthesized speech token was a [ba] or a [da] (see below for details) and responded accordingly. In the second task, subjects heard a pure tone token and judged whether it was rising or falling in pitch. Each task was performed in two attentional conditions. In a binaural condition, the speech or tonetoken was played simultaneously to both ears. In a dichotic condition, the speech token was played to one ear and the tone token to the other; (the ear to which the rel evant token was played was randomized from trial to trial). I n one case, subjects attended to the speech and made the [ba]/[da] judgment. In the other case, they attended to the tone and made the pitch judgment. In the dichotic conditions, subjects were required to attend to one message while ignoring or inhibiting the other, thereby maximizing demands on auditory selective attention. However, decision and response execution are comparable in both the binaural and dichotic conditions.

The primary prediction is that posterior (parietal) activation will discriminate the binaural and dichotic conditions more than will anterior (cingulate) activation. One subsidiary prediction is that speech processing will be more associated with left hemisphere sites and pitch with right hemisphere sites (Zatorre et al., 1991). Within-hemisphere differences in speech and pitch localization are also anticipated. Further, differences in the relative activation of regions associated with the attended-to and ignored stimuli should be observed as well.

\section{Method}

Subjects. Twenty-five neurologically normal adult subjects (12 males and 13 females) participated in this study and were reimbursed for their participation. None had any history of language impairment or known auditory problems.

Speech materials. The speech stimuli used in the perception task were four synthetic syllables-two [da] and two [ba]. The speech was synthesized with the serial formant synthesizer of Haskins Laboratories. Each syllable was $240 \mathrm{~ms}$ in duration, with a falling F0 from 100 to $95 \mathrm{~Hz}$ and a constant amplitude. The frequencies and bandwidths of the formants were ramped through the first $40 \mathrm{~ms}$ and held constant thereafter. Only the second formant transition (first 40 $\mathrm{ms}$ ) differed between the stimuli. In all stimuli, $\mathrm{F} 1$ rose from $450 \mathrm{~Hz}(60 \mathrm{~Hz}$ bandwidth) to $700 \mathrm{~Hz}(130 \mathrm{~Hz}$ bandwidth); F 3 rose from $2400 \mathrm{~Hz}$ ( $150 \mathrm{~Hz}$ bandwidth) to $2600 \mathrm{~Hz}$ ( $160 \mathrm{~Hz}$ bandwidth). The bandwidth for F2 was ramped from 90 to $70 \mathrm{~Hz}$. The $\mathrm{F} 2$ transition varied for each of the four tokens. For the two [ba] tokens, it 
rose from 900 to $1200 \mathrm{~Hz}$ and from 1000 to $1200 \mathrm{~Hz}$. For the two [da] tokens, the $\mathrm{F} 2$ transition fell from 1500 and $1600 \mathrm{~Hz}$ to $1200 \mathrm{~Hz}$.

Tone materials. The four tone stimuli were pure tones synthesized using the sine wave synthesizer of Haskins Laboratories. Two of the tones were falling in pitch and two rising. The frequency of the falling tones was interpolated linearly from 1900 to $1200 \mathrm{~Hz}$ and from 1800 to $1300 \mathrm{~Hz}$. The rising tones covered the same ranges moving in the opposite direction, i.e., from 1200 to $1900 \mathrm{~Hz}$ and 1800 to $1300 \mathrm{~Hz}$. The tones were $240 \mathrm{~ms}$ in duration.

Noise bursts. Finally, a broad band noise burst of $240 \mathrm{~ms}$ duration was synthesized using the software package Sound Edit Pro. This sound was similar to static from a radio or television. These tokens were used for the baseline/control condition of the present study.

Procedure Thegeneral procedure involved four runs of approximately $5.5 \mathrm{~min}$ in which experimental tasks (speech only, pitch only, speech dichotic, pitch dichotic) were interleaved with a common control task (noise burst detection). Tasks were interleaved such that 10 noise burst detection trials (40 s) were followed by 10 experimental trials ( $40 \mathrm{~s}$ ), then 10 noise trials, etc. For instance, in the speech-only run, the speech-only task was interleaved with the noise burst detection task such that each was repeated four times. Similar alternations were used for the other three experimental tasks. In all conditions, theinterstimulus interval was $4 \mathrm{~s}$. For the initial 13 subjects, response time collection procedures were not yet in place. For these subjects responses to stimuli were made using a bulb that made a clicking sound in the control room when pressed. In the noise burst control, subjects responded with a squeeze each time a token was heard. In the experimental tasks, subjects responded if the stimulus was a [ba] (in the speech task) or if the tone had a rising pitch (in the pitch task). For the subsequent 12 subjects, an on-line response time collection procedure using a fiber optic two-channel box was in place to record responses (left button for [ba], rising pitch, or noise; right button for [da] or falling pitch). For these subjects, stimuli were played directly from a Mad ntosh II computer that also recorded response times and accuracy. ${ }^{1}$ I $n$ all tasks, the stimuli were presented in pseudo-random order, and in

\footnotetext{
1 The differences in response procedures for the earlier $(\mathrm{N}=13)$ and later $(\mathrm{N}=12$ ) subjects should factor out since the critical repeated measures comparisons are between tasks and Attention Condition and for any subject, response procedures across conditions were comparable. Nonetheless, we initially analyzed the data to determine whether any of the critical effects or interactions were qualified by this response type difference by grouping subjects accordingly. No main effect of response type was observed nor were any of the reported effects or interactions qualified by this factor. Hence, in all reported results, we have collapsed the two groups.
}

the dichotic tasks, the ear receiving the speech stimuli was also varied pseudo-randomly. In the first task, subjects heard the speech syllables presented binaurally, and in the second task, subjects heard the tones binaurally. In the third task, subjects heard a tone in one ear and a syllable in the other (randomized from token to token) and were instructed to ignore the tones and respond only to speech stimuli. Finally, in the fourth task, subjects again heard a tone in one ear and a syllable in the other, but were instructed to ignore the speech and respond only to the tone.

Prior to starting the experiment, each subject listened to a practice tape that included examples of the noise burst, the speech tokens, and the tone tokens presented both binaurally and dichotically. They heard this tape in the experiment room, using the experiment headphones. Subjects were given a printed key to follow that identified each stimulus as a [ba] or [da] or as "rising" or "falling." Subjects were all owed to listen to the practice tape several times if they so chose. Generally, if more than one repetition of the practice tape was requested, it was in order to repeat the speech stimuli, which were harder to identify than the tone stimuli. The subjects were wearing well-insulated headphones and were instructed to close their eyes during thetasks. The entire experiment lasted approximately $1 \frac{1}{2} \mathrm{~h}$.

Imaging. Imaging was performed on a 1.5 Tesla (General Electronics,) MR imaging system equipped with echo planar imaging (EPI) hardware from Advanced NMR (Willmington, MA). Subjects' heads were immobilized within a circularly polarized head coil by using a neck support, foam wedges, and a restraining band drawn tightly around the forehead. Conventional spin echo sagittal $\mathrm{T}_{1}$-weighted (TE $=11 \mathrm{~ms}$, TR $=667$ $\mathrm{ms}, \mathrm{FOV}=24 \mathrm{~cm}$, slice thickness $=5 \mathrm{~mm}$, slice gap $=0.0 \mathrm{~mm}, 256 \times 192 \times 1 \mathrm{Nex}$ ) localizer scans were first obtained from which five axial-oblique images were prescribed $(\mathrm{TE}=500 \mathrm{~ms}, \mathrm{TR}=13 \mathrm{~ms}$, $\mathrm{FOV}=40 \times 20 \mathrm{~cm}, 256 \times 192 \times 2 \mathrm{Nex}, 8-\mathrm{mm}$ section thickness) parallel to the intercommisural line. These slices were centered along the vertical (z) axis of Talairach and Tournoux (1988) at $z=+1,+12,+24$, $+36,+48$ and were used as anatomic images on which to overlay activation maps. Activation images were collected using an EPI gradient echo sequence (flip angle $60^{\circ}, \mathrm{TE}=45 \mathrm{~ms}, \mathrm{TR}=1700 \mathrm{~ms}, \mathrm{FOV}=40 \times 20$ $\mathrm{cm}, 128 \times 64 \times 1$ Nex, 8-mm section thickness) in the five slice locations described above. Two-hundred images per slice location were collected while the subject performed each of the four on conditions (pitch binaural, speech binaural, pitch dichotic, and speech dichotic) in alternation with the off (noise burst task) condition. Each task and control was repeated four times in alternation over the course of one series.

Initial data analysis. Data analysis was performed using software written in MATLAB (MathWorks, 
Natick, MA). One hundred seventy-six images of the 200 images per slice in a series were analyzed; 88 images obtained during the task condition were compared with 88 images obtained during control condition. Twenty-four images of a series were discarded at the 8 transition periods (beginning and end of task and control) to account for temporal variation of hemodynamic changes that occur during these periods. The 176 images from each series were median filtered. Before median filtering, the temporal mean intensity image was subtracted from each acquisition and then readded after filtering. Activated pixels were detected for each pair of activation tasks using a Student's t test. If the $t$ value for a given pixel was above 2.0 the pixel was considered activated, and the sum of these activated pixels was computed for each subject in each region of interest (ROI). For normally distributed data using a t test, $\mathrm{t}>2$ corresponds to $\mathrm{P}<.05$. This threshold for activation provides a fairly conservative criterion for identifying task-related activity from other sources of signal variation. Images were cluster filtered so that isolated activated pixels without at least two activated neighbors were discarded. Subject head movements were analyzed but not corrected. The motion of center of mass was less than 0.1 pixel for most subjects and never more than 0.5 pixel. Calculated t maps did not show substantial rim artifacts or apparent activation at edges, confirming that head movements were negligible.

On each anatomic and functional image of each subject, the position of the anterior and posterior commissures and right and left brain edges and the direction of the midline were found manually. These reference points created five scaling factors: $x l$ for midline to left brain edge; xr for midline to right brain edge; y1 for anterior brain edge to anterior commissure $(A C)$ : y2 for AC to posterior commissure (PC); and y3 for $\mathrm{PC}$ to posterior brain edge. Each anatomical image and corresponding activation map were coregistered to twodimensional standard Talairach form using cubic proportional fitting, a piecewise warping algorithm based on the scaling factors. This divided the brain into rectangular volumes defined by upper- and lowercase letters and by $x, y, z$ coordinates of the standard Talairach coordinate system. A composite anatomic image for each slice location was obtained by adding Talairach-scal ed anatomical images from each of the 25 subjects. A composite activation map of the 25 subjects was made at each slice location and for each of the four experimental tasks and overlaid on the composite anatomic slice. Activated pixels with highest median $t$ values ( $\mathrm{t}>0.50)$ are shown in color, Fig. 8.

Regions of interest. Nine primary ROIs were examined (Table 1) along with other regions in subsequent analyses. We chose ROIs that have been implicated either in previous studies of auditory processing or in previous studies of attention: prefrontal, inferior frontal, anterior cingulate, posterior cingulate, superior temporal, middle temporal, inferior parietal, superior parietal, and precuneus. The full extent of ROI was defined by rectangular volumes that encompassed wellestablished gyral and sulcal patterns and Brodmann's areas as defined in the stereotaxic atlas of Talairach and Tournoux. Cortex within ROI was approximated by coordinates referenced to $x, y$, and $z$ axes (see Table 1 for Brodmann's areas and Talairach coordinates). Regions were defined on composite anatomic images and then applied to individual subjects. The rectangular volumes were large enough to account for anatomic variability between subjects (Andreasen et al., 1994) and to insure reproducible coverage of areas of interest in a large number of subjects. These volumes also permit demarcation of areas based on both anatomy and Brodmann's areas. Intensity thresholding eliminated spurious activations outsi dethe brain from entering into statistical analysis so that rectangular volumes could be chosen to include areas of peripheral gray matter even though a portion of the volume extended beyond the brain.

Statistical analyses. The first stage of data analysis, described above, provides activation data (t maps) for each subject in each of the four experimental conditions (pitch binaural, speech binaural, pitch dichotic, and speech dichotic) relative to the noise burst detection control task. Analyses of variance (ANOVA) were performed on these data for each region of interest using subjects as the unit of analysis with the following independent variables: Hemisphere, Task (speech vs pitch), and Attention Condition (binaural vs dichotic). In the omnibus analysis, ROI was also included as an independent variable. Each of these were repeated measures variables. The primary dependent variable was the number of pixels within these regions of interest that demonstrated positive changes in MR image intensity (by t test) between tasks being compared. Hence, these analyses allowed us to determine whether specific differences in the mean numbers of activated pixels for different tasks were reliable across subjects. Initially, an omnibus ANOVA was conducted, which included as a factor the nine ROI s in order to test for Attention Condition or Task interactions with ROI . Subsequently, we examined specific regions of interest and divided these into meaningful subregions where relevant, in order to provide a more fine-grained picture of the effects of attention condition or task. Both reaction time and error data obtained on 12 subjects (see Method) were also analyzed using the following repeated measures variables: Attention Condition and Task. The results from these behavioral analyses are presented first. 
TABLE 1

Regions of Interest

\begin{tabular}{|c|c|c|c|c|c|c|c|}
\hline \multirow{2}{*}{$\begin{array}{r}\text { Region } \\
\text { 1. Prefrontal }\end{array}$} & \multirow{4}{*}{$\begin{array}{l}\text { Gyrus } \\
\text { Transverse frontopolar and } \\
\text { anterior aspect of superior } \\
\text { frontal gyrus and middle } \\
\text { frontal gyrus }\end{array}$} & \multirow{2}{*}{$\begin{array}{c}\text { Brodmann area } \\
\text { 8 } \\
9\end{array}$} & \multicolumn{5}{|c|}{ Talairach coordinates ${ }^{a}(x / y / z)$} \\
\hline & & & $\begin{array}{l}1.0 / 3.9 / 48 \\
4.3 / 4.4 / 24\end{array}$ & $0.3 / 3.0 / 48$ & $2.6 / 3.0 / 48$ & & \\
\hline & & & $0.9 / 5.5 / 36$ & $2.7 / 4.4 / 36$ & $3.4 / 3.8 / 36$ & $2.9 / 2.3 / 36$ & $4.1 / 2.6 / 36$ \\
\hline & & 10 & $0.5 / 5.9 / 1.0$ & 2.3/6.7/1.0 & $3.5 / 5.7 / 1.0$ & & \\
\hline & & & $0.5 / 5.9 / 12$ & $2.2 / 6.7 / 12$ & $3.7 / 6.0 / 12$ & $4.3 / 4.5 / 12$ & \\
\hline & & & $0.4 / 6.0 / 24$ & $2.3 / 5.5 / 24$ & $2.3 / 4.5 / 24$ & & \\
\hline \multirow[t]{3}{*}{ (Dorsolateral) } & Anterior half of the inferior & 46 & 4.7/4.8/1.0 & & & & \\
\hline & frontal sulcus: bordered by & & 5.3/3.3/12 & & & & \\
\hline & $\begin{array}{l}\text { inferior aspect of middle } \\
\text { frontal gyrus and by ante- } \\
\text { rior aspect of pars triangu- } \\
\text { laris of the inferior frontal } \\
\text { gyrus }\end{array}$ & & $4.5 / 2.2 / 24$ & $5.4 / 4.4 / 24$ & & & \\
\hline \multirow[t]{2}{*}{ 2. Inferior frontal } & Inferior frontal gyrus: pars & 44 & $0.8 / 1.1 / 12$ & $5.5 / 0.8 / 12$ & $4.6 / 0.1 / 24$ & $5.3 / 1.1 / 24$ & \\
\hline & $\begin{array}{l}\text { opercula and all of pars } \\
\text { triangularis, except its } \\
\text { anterior aspect }\end{array}$ & 45 & $4.7 / 2.5 / 1.0$ & $5.5 / 2.5 / 1.0$ & $4.3 / 2.1 / 12$ & $5.5 / 2.3 / 12$ & \\
\hline \multirow[t]{2}{*}{ 3. Superior temporal } & Superior temporal gyrus & 22 & $\begin{array}{l}4.6 / 0.5 / 1.0 \\
6.3 /-3.6 / 12\end{array}$ & $\begin{array}{l}4.9 /-0.3 / 1.0 \\
6.0 /-5.1 / 12\end{array}$ & $4.5 /-1.0 / 1.0$ & $6.2 /-2.0 / 1.0$ & \\
\hline & & 42 & $5.8 /-1.2 / 12$ & $6.0 /-2.2 / 12$ & & & \\
\hline \multirow[t]{2}{*}{ 4. Middletemporal } & Middle temporal gyrus & 21 & $6.3 /-2.1 / 1.0$ & $5.9 /-5.4 / 1.0$ & & & \\
\hline & & 39 & $4.6 /-7.8 / 24$ & & & & \\
\hline \multirow[t]{2}{*}{ 5. Anterior cingulate } & Anterior cingulate gyrus & 24,32 & 0.3/3.9/1.0 & $0.3 / 3.6 / 12$ & $0.2 / 2.1 / 24$ & & \\
\hline & & & $0.3 / 0.7 / 36$ & $0.3 /-0.7 / 36$ & $0.3 /-1.2 / 36$ & & \\
\hline \multirow[t]{2}{*}{ 6. Posterior cingulate } & Posterior cingulate gyrus & 23 & $0.3 /-3.7 / 24$ & & & & \\
\hline & & & $0.3 /-20 . / 36$ & & & & \\
\hline \multirow[t]{3}{*}{ 7. Inferior parietal } & Supramarginal gyrus and & 39,40 & $6.2 /-3.8 / 24$ & $5.4 /-5.2 / 24$ & & & \\
\hline & angular gyrus & & $5.9 /-3.4 / 36$ & $5.8 /-5.0 / 36$ & $4.7 /-6.9 / 36$ & & \\
\hline & & & $4.9 /-3.6 / 48$ & $4.5 /-5.0 / 48$ & & & \\
\hline 8. Superior parietal & Superior parietal Iobule & 7 & $2.7 /-6.5 / 48$ & & & & \\
\hline \multirow[t]{2}{*}{ 9. Precuneus } & Precuneus (medial aspect of & 7 & $0.3 /-6.0 / 36$ & $0.3 /-7.1 / 36$ & & & \\
\hline & parietal lobe) & & $0.3 /-4.6 / 48$ & $0.3 /-6.6 / 48$ & & & \\
\hline
\end{tabular}

a Coordinates approximate center of cortex. x coordinates are positive (right hemisphere) and negative (left hemisphere).

\section{RESULTS}

Behavioral Analyses

In the latency (RT) analysis (for the 12 subjects for whom such measures were available), a main effect of Attention Condition was obtained, $F(1,11)=10.57$, $\mathrm{P}<.01$. The mean latencies were $878 \mathrm{~ms}$ for the binaural conditions and 1019 ms for the dichotic conditions. The main effect of Task and the interaction between Attention Condition and Task were not significant. In the error rate analysis, there was a main effect of Attention Condition, $\mathrm{F}(1,11)=10.40, \mathrm{P}<.01$ with mean error rates of 17 and $26 \%$ in the binaural and dichotic conditions, respectively. There was also a main effect of Task, $\mathrm{F}(1,11)=17.99, \mathrm{P}<.01$. The mean error rates were $12 \%$ for the pitch judgment and $32 \%$ for the speech. The interaction was not significant. Hence, both latency and accuracy analyses suggest that the dichotic conditions were more difficult, while the error analysis further suggests that the speech judgment task was somewhat more difficult than the pitch judgment task.

\section{Omnibus Analysis of fMRI Data}

This analysis consisted of the following factors: ROI (nine regions), Attention Condition (binaural vs dichotic), Task (speech vs pitch), and Hemisphere. A significant main effect of Attention Condition was obtained, $F(1,24)=6.04, P<.025$. The mean number of pixels activated in the binaural condition was 63 , while the mean for the dichotic condition was 77. However, this main effect was strongly qualified by $\mathrm{ROI}$ as revealed in an Attention Condition $\times$ ROI interaction, $F(8,192)=$ 4.02, $\mathrm{P}<.001$. As indicated in Fig. 1, significant increases in activation from binaural to dichotic conditions were obtained only for the superior temporal, inferior frontal, and inferior parietal ROIs. Of note, the activation in the anterior cingulate gyrus actually decreased nonsignificantly. A significant main effect of hemisphere was obtained, $\mathrm{F}(1,24)=11.25, \mathrm{P}<.01$. The mean numbers of activated pixels were 77 and 63 for the right and left hemispheres, respectively. An Attention Condition $\times$ Task interaction was obtained, 


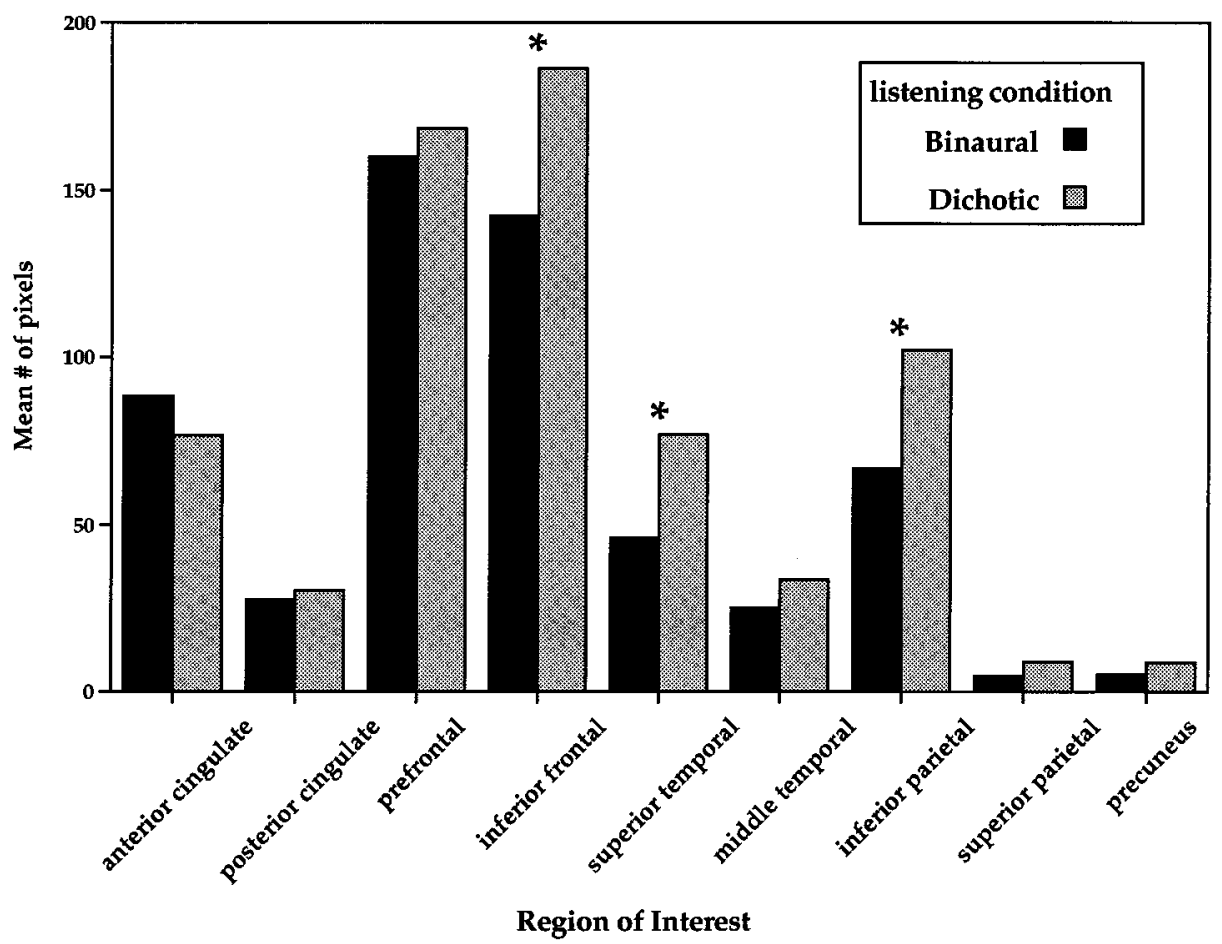

FIG. 1. Attention Condition $\times$ ROI interaction for nine ROI $s$ in the Omnibus analysis.

$F(1,24)=8.41, P<.01$. Means of 55 and 82 were obtained for the binaural and dichotic speech tasks, respectively, while means of 71 and 71 were obtained for the binaural and dichotic pitch tasks. However, this global effect showing greater increases in speech but not pitch in the dichotic condition was qualified by a marginally significant Attention Condition $\times$ Task $\times$ ROI interaction, $F(8,192)=1.82, P=.076$. This qualification of the Attention Condition $\times$ Task interaction by ROI is examined carefully in the separate ROI analyses detailed bel ow.

\section{Region of Interest Analyses}

Nine broad regions were included in the Omnibus ANOVA (anterior cingulate, posterior cingulate, prefrontal, inferior frontal, superior temporal, middle temporal, inferior parietal, superior parietal, and precuneus). Given the various interactions (e.g., Attention Condition $\times$ ROI and Attention Condition $\times$ Task $\times$ ROI) and, further, given that several of these ROIs can be meaningfully further divided into coherent subregions, we conducted a number of more specific analyses for these more finely aut regions. We begin with the three ROIs where significant effects of attention condition were obtained in the Omnibus analysis and then describe rel evant effects and interactions from the other ROIs.

Superior temporal gyrus. The region was divided into two coherent subregions ([B.A.] 22 vs. [B.A.] 42). A main effect of Attention Condition was observed in this region, $F(1,24)=12.02, \mathrm{P}>.01$. The means for the binaural and dichotic conditions were 23 and 38, respectively. This effect was qualified, however, by an interaction with subregion, $F(1,19=19.33, P<.001$. This interaction, shown in Fig. 2, reveals that the increase from binaural to dichotic conditions was confined to [B.A.] 22 within the STG and had no influence on [B.A.] 42. ${ }^{2}$ These results were not further qualified by interactions with task, or by hemisphere (both Fs $<1.0$ ).

Inferior frontal gyrus. This ROI was divided into two subregions ([B.A.] 44 and [B.A.] 45). A main effect of hemisphere is seen, $\mathrm{F}(1,24)=6.07, \mathrm{P}<.025$. The means for $\mathrm{LH}$ and $\mathrm{RH}$ were 55 and 67, respectively. A main effect of Attention Condition was found, $F(1,24)=$

\footnotetext{
${ }^{2}$ One of the reviewers of the manuscript raised the question of whether primary auditory cortex [B.A.] 41 would show any modulation of activation levels as a function of the attentional manipulation. We had not included this region in the study because we assumed that activation of this region would be nearly equivalent in the experimental and the control (noise burst detection) conditions and therefore would cancel out in the subtractions. Examination of this region confirmed that expectation. The majority of subjects had scores of 0 pixels in this region, and given this extreme skew to 0 statistical tests would be inappropriate; in any event, the means for the binaural and dichotic conditions were 1.3 and 2.9 pixels, respectively. Clearly, the question of whether selective attention modualtes primary sensory regions is an important one but we must conclude that the current experimental design does not allow us to adequately target this region.
} 


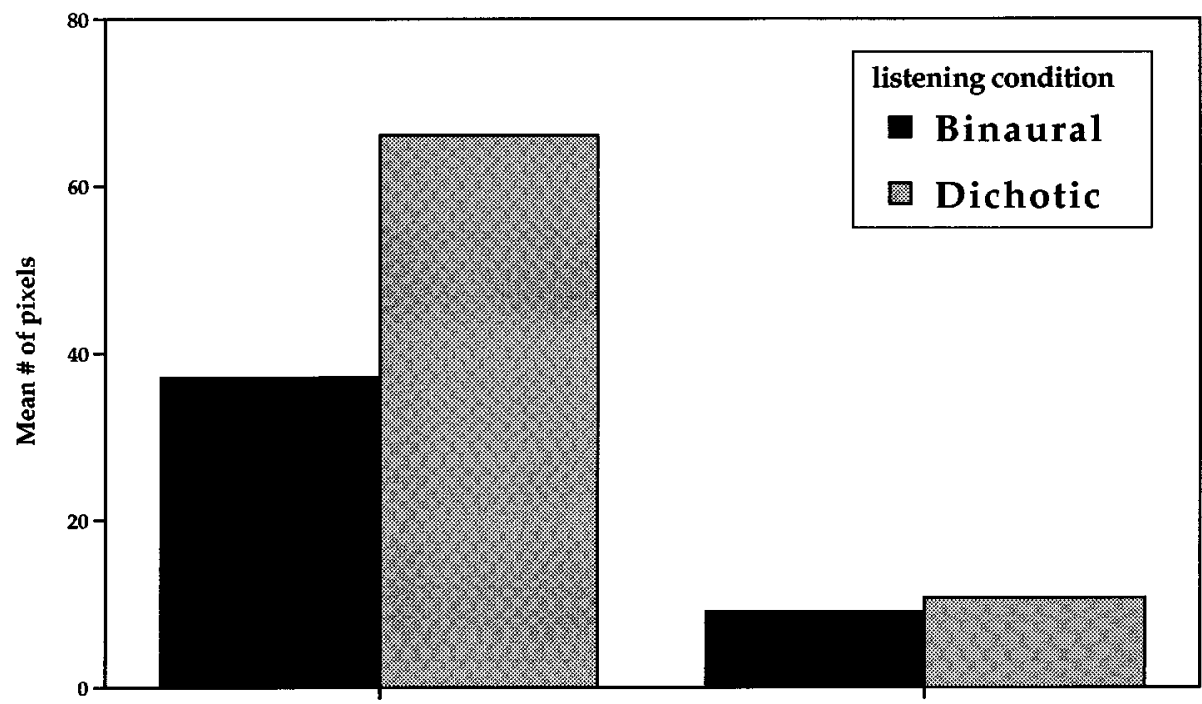

BA22

BA42

\section{Superior Temporal Gyrus}

FIG. 2. Attention Condition $\times \mathrm{ROI}$ interaction in the superior temporal gyrus.

$6.95, \mathrm{P}<.025$. The means for the binaural and dichotic conditions were 53 and 69, respectively. However, this effect was qualified by an interaction with subregion, $F(1,24)=4.29, P<.05$. This two-way interaction was, in turn, qualified by hemisphere, $F(1,24)=5.09, P<$ .05. This three-way interaction, shown in Fig. 3, reveals that the increase from binaural to dichotic conditions was observed primarily in [B.A.] 44 and here, only in the right hemisphere. Whilethere was no main effect of
Task there was a reliable Task $\times$ Subregion $\times$ Hemisphere interaction, $F(1,24)=4.37, P<.05$. As shown in Fig. 4, this interaction reveals that within [B.A.] 44, speech was associated with equivalent $\mathrm{LH}$ and $\mathrm{RH}$ activation, while pitch, by contrast, was associated with relatively greater $\mathrm{RH}$ activation. These two informative three-way interactions were not qualified by a four-way interaction $(F<1.0)$. Hence, it is clear that the RH [B.A.] 44 increase in the dichotic condition and

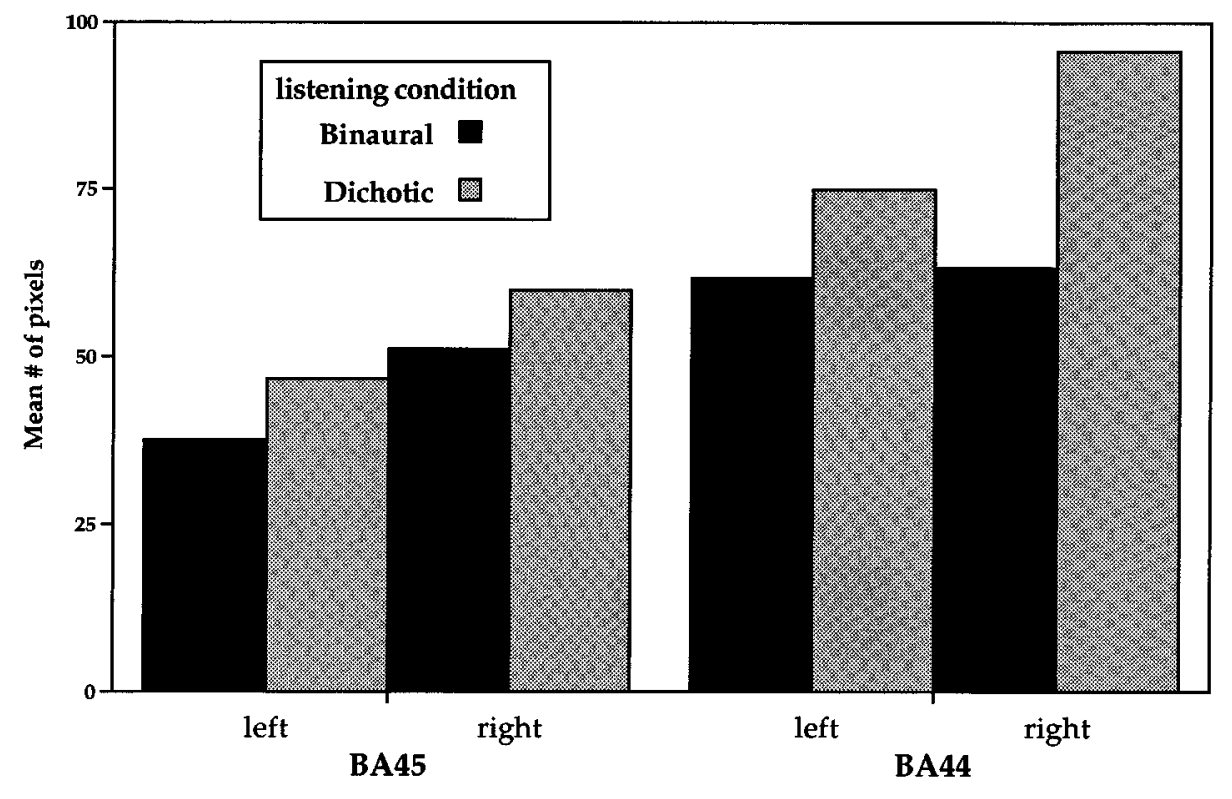

Inferior Frontal Gyrus

FIG. 3. Attention Condition $\times \mathrm{ROI} \times$ Hemisphere interaction in the inferior frontal gyrus. 


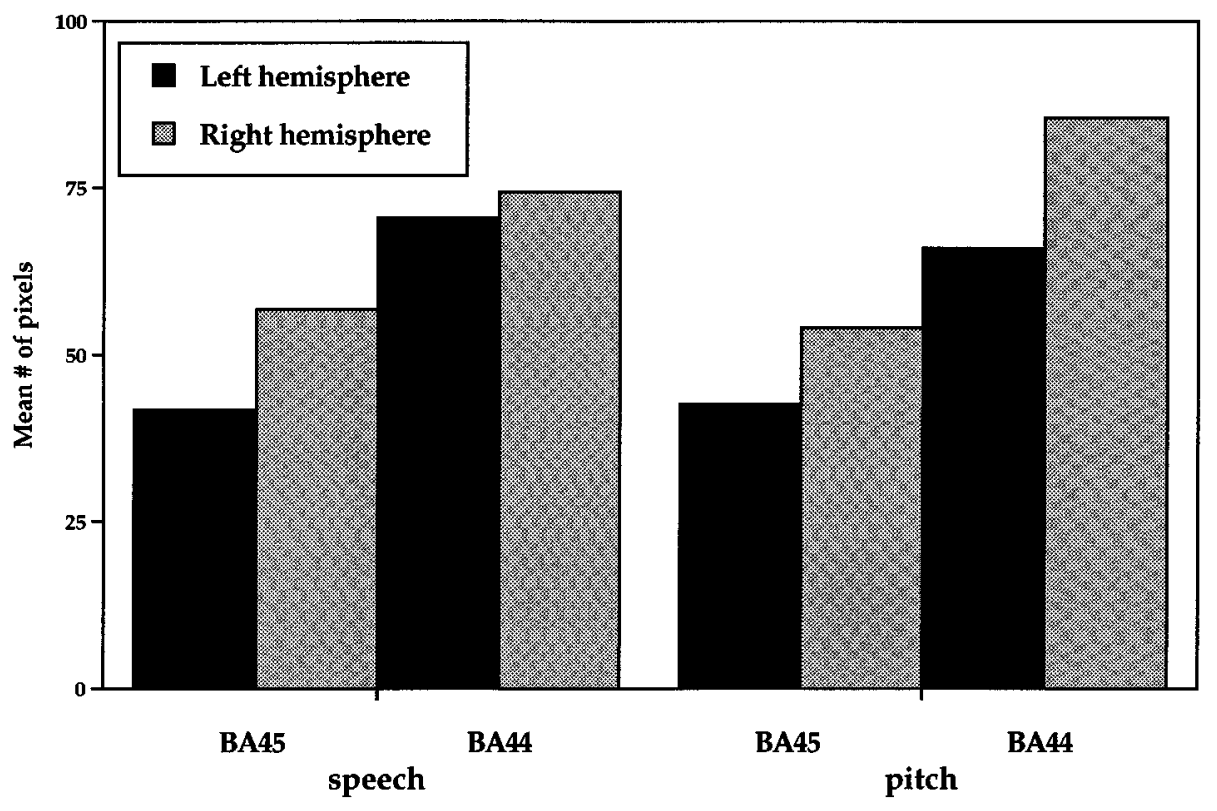

Inferior Frontal Gyrus

FIG. 4. Task $\times$ ROI $\times$ Hemisphere interaction in the inferior frontal gyrus.

the differential use of the $\mathrm{LH}$ and $\mathrm{RH}$ by speech and pitch were statistically independent.

Recently Shaywitz et al. (1995) reported a robust sex by hemisphere interaction at sites within the inferior frontal gyrus in a rhyme judgment task on visually presented pseudoword stimuli. Males $(\mathrm{N}=19)$ showed a substantially $\mathrm{LH}>\mathrm{RH}$ activation pattern. By contrast, females ( $\mathrm{N}=19$ ), on average, showed a bilateral $(\mathrm{LH}=\mathrm{RH})$ activation pattern. This relatively stronger $\mathrm{RH}$ involvement for females led us to ask whether a similar sex by hemisphere interaction will be obtained for spoken language materials employed in the present study. Analyses revealed a reliable sex by hemisphere interaction for the speech judgment task at (B.A.) 45, $\mathrm{F}(1,23)=5.27, \mathrm{P}<.05$. For males the $\mathrm{LH}$ and $\mathrm{RH}$ means were 47 and 40 , respectively. For females the $\mathrm{LH}$ and $\mathrm{RH}$ means were 24 and 57, respectively. I mportantly, the sex by hemisphere interaction was not found on the pitch judgment task in this region $(\mathrm{F}<1.0)$.

Inferior parietal lobe In the inferior parietal lobea strong main effect of Attention Condition was obtained, $F(1,24)=15.976, P<.001$, with means of 67 and 102 for the binaural and dichotic conditions, respectively. Of note is the fact that in this region the effect is not qualified by Task. It is evident that in general the effect is greater in the RH (63 to 107) than in the $\mathrm{LH}$ (70 to 96); however, this interaction failed to reach significance $(F(1,24)=2.58, P=.12)$.

Prefrontal. The prefrontal region was divided into three distinct subregions ([B.A.] 10, [B.A.] 9, and [B.A.]
8). A main effect of hemisphere was obtained, $F(1,24)=$ 14.08, $\mathrm{P}<.001$. The means for $\mathrm{LH}$ and $\mathrm{RH}$ were 63 and 47 pixels, respectively. An Attention Condition $\times$ Task interaction was observed, $\mathrm{F}(1,24)=12.97, \mathrm{P}<.01$. This crossover interaction, shown in Fig. 5, reveals that for speech, an increase from binaural to dichotic conditions was observed, while for pitch, a decrease was observed. The increase in speech was significant in subsequent contrasts, $\mathrm{F}(1,24)=8.79, \mathrm{P}<.01$, and similarly the decrease in pitch was also reliable, $F(1$, $24)=4.54, P<.05$. These effects and interactions were not further qualified by any higher-order interactions.

Prefrontal dorsolateral region. Brodmann's Area 46 has been much examined in language, working memory, and attention tasks. We isolated this region for a separate analysis. A main effect of Attention Condition was observed, $\mathrm{F}(1,24)=7.81, \mathrm{P}<.025$, with means of 36 and 48 for the binaural and dichotic tasks, respectively. The only other significant effect was hemisphere, $F(1,24)=8.0, P<.01$, with means of 33 and 50 in the $\mathrm{LH}$ and $\mathrm{RH}$, respectively.

Middle temporal gyrus. This region was divided into two subregions ([B.A.] 21 vs. [B.A.] 39). In this region, no main effects of Task or Attention Condition were observed. However, a significant three-way interaction between Attention Condition, Task, and Hemisphere was obtained, $\mathrm{F}(1,24)=4.43, \mathrm{P}<.05$. An inspection of the means plotted in Fig. 6 indicates that relatively larger increases from binaural to dichotic conditions for speech were seen in the LH than in the 


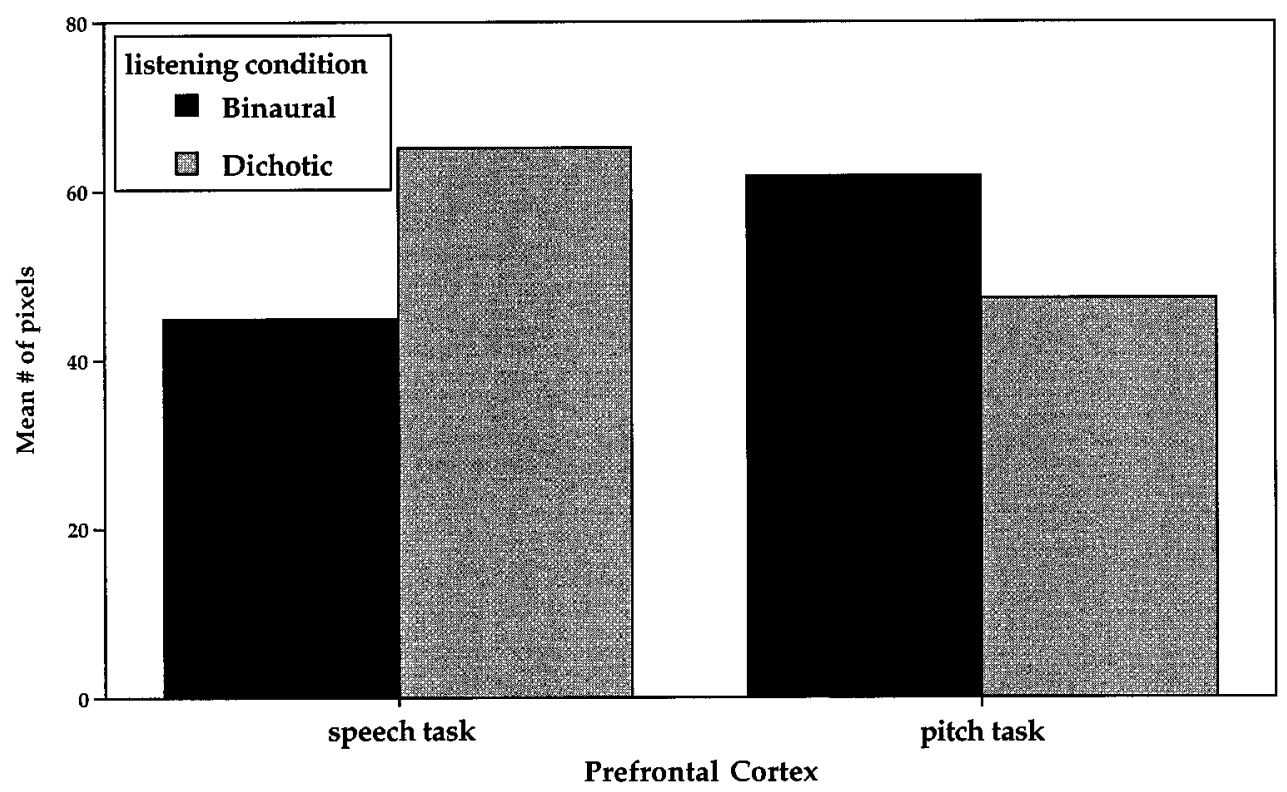

FIG. 5. Attention Condition $\times$ Task interaction in the prefrontal cortex.

$\mathrm{RH}$, while for pitch the increase is limited to the $\mathrm{RH}$. No other interactions were significant.

Superior parietal lobule and precuneous. As noted above, these two regions failed to show a significant effect of Attention Condition in the Omnibus ANOVA. However, in the separate ROI analyses, both sites showed a numerically small, though significant, main effect of Attention Condition. For the superior parietal lobule, an $F(1,24)=4.46(P<.05)$ obtained, with means of 5 and 9 in the binaural and dichotic conditions, respec- tively. In the precuneus a similar pattern obtained, $F(1,24)=4.31, P<.05$. Further, in the precuneus there was a reliableAttention Condition $\times$ Hemisphere interaction, $\mathrm{F}(1,24)=4.35, \mathrm{P}<.05$, which is shown in Fig. 7 . The effect in this $\mathrm{RO}$ appeared to be due to greater $\mathrm{RH}$ than LH increases in activation. Given the absence of main effects of Attention Condition in the initial Omnibus analysis, the main effects here should be viewed cautiously, though the Attention Condition $\times \mathrm{Hemi}-$ sphere interaction in the precuneus is noteworthy.

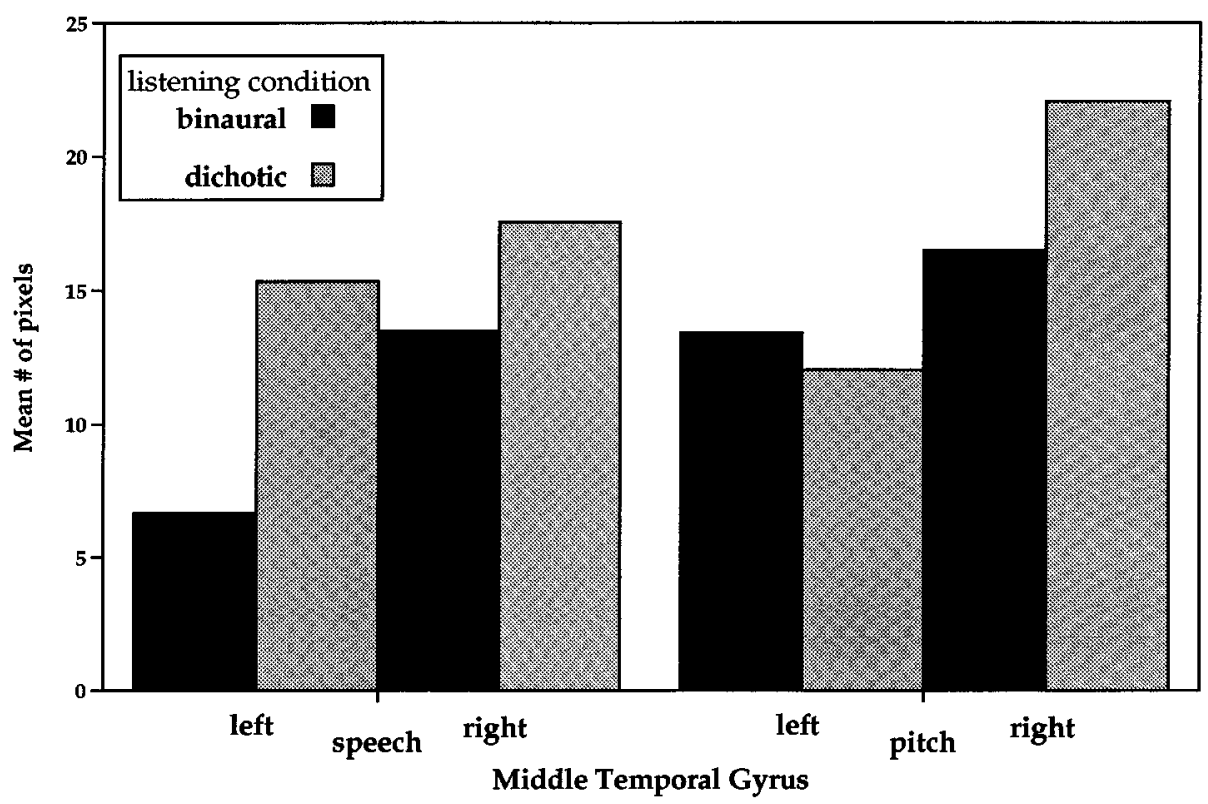

FIG. 6. Attention Condition $\times$ Task $\times$ Hemisphere interaction in the middle temporal gyrus. 


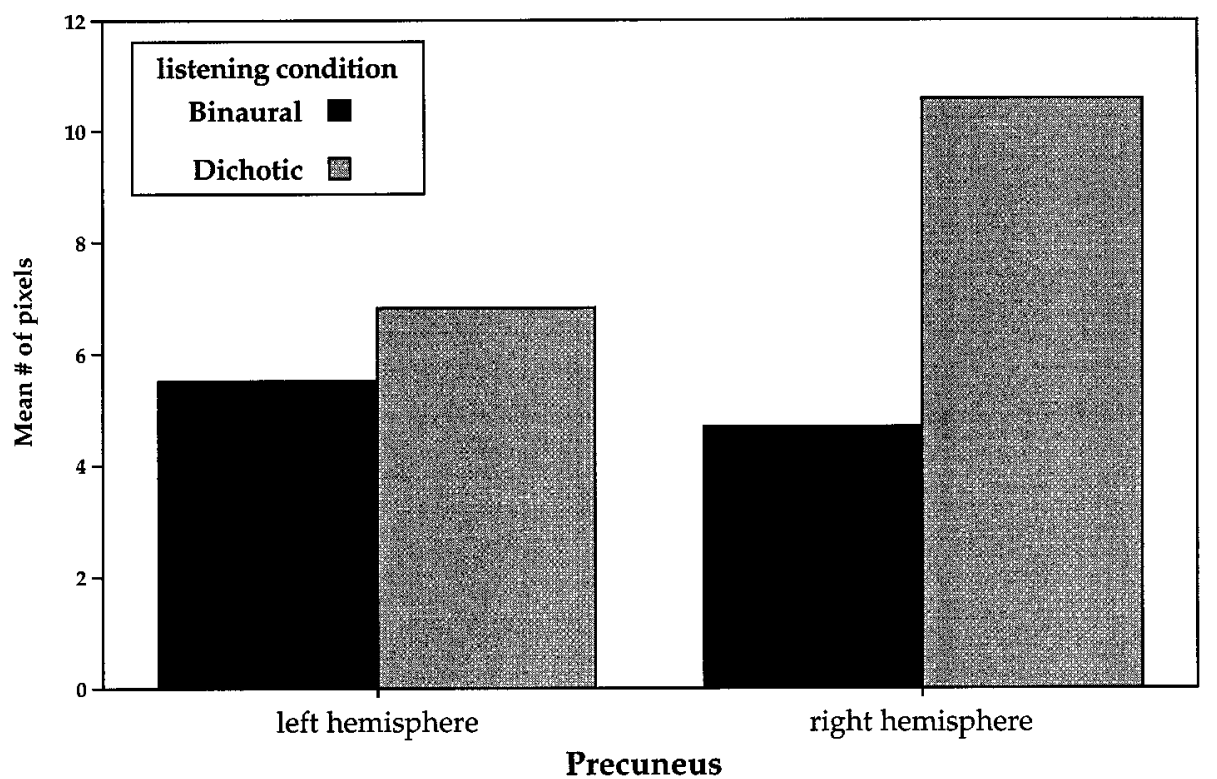

FIG. 7. Attention Condition $\times$ Hemisphere interaction in the precuneus.

Composite image Composite activation images for the 25 subjects (revealing pixels with median $t$ values $>.50$ ) are shown in Fig. 8. The pattern of activations seen in these images is consistent with the main findings from the ROI analyses described in the preceding section. ${ }^{3}$ Clear evidence of increased spatial extent of activation in the two dichotic conditions relative to the two binaural conditions is seen in the inferior frontal gyrus, superior temporal gyrus, and inferior parietal lobule. Therelative increase from speech binaural to speech dichotic conditions within the prefrontal region is evident as well. Increased $\mathrm{LH}$ activation in both speech conditions relative to both pitch conditions is seen in Broca's Area ([B.A.] 44) within the inferior frontal gyrus. Finally, no changes within the anterior cingulate gyrus are seen in these composites.

\footnotetext{
${ }^{3}$ The activation composites converged with the basic patterns evident in the ROI analyses. It should be noted that the composite analysis will not necessarily mirror the ROI analyses in all respects. This is because the ROI analysis measures the number of activated pixels in a given condition within a broadly defined ROI, but does not require that these activated pixels be at identical locations within the region for all subjects. The composite, on the other hand, examines activation levels across subjects for a given pixel. Both analyses are informative. If a ROI responds to a certain subtraction condition with, for instance, increased numbers of activated pixels on average, but if there is intersubject variability in the exact locations of these pixels, then the composite analysis might not reflect this increase, while the ROI analysis most certainly will. Only when the activated sites are homogeneous (across subjects) will this be apparent in the composite. We must allow for some variability across subjects within a given cortical region, and the ROI analyses are thus important. However, if we also want to determine which specific pixels are most commonly activated, then the composite image is informative. In any event, in the present experiment the two types of analyses yielded comparable findings.
}

The composite image also revealed differences in activation from binaural to dichotic conditions in the superior aspect of the prefrontal region ([B.A.] 6), particularly in the $\mathrm{RH}$. This region was not among the primary sites examined in the ROI analyses. However, given the activation seen in the composite we conducted an ROI analysis on this region to test for statistical significance. A main effect of Attention Condition was seen, $\mathrm{F}(1,24)=7.18, \mathrm{P}<.025$. The relevant mean number of pixels were 6 and 10 in the binaural and dichotic conditions, respectively. A main effect of hemisphere was also obtained, $\mathrm{F}(1,24)=6.92$, $\mathrm{P}<$ .025 , with RH and LH means of 11 , and 6 , respectively. Several two-way interactions were observed. Attention Condition by Task, $\mathrm{F}(1,24)=10.74, \mathrm{P}<.01$, Attention Condition by Hemisphere, $\mathrm{F}(1,24) 4.57, \mathrm{P}<.05$, and Task by Hemisphere, $\mathrm{F}(1,24)=4.63, \mathrm{P}<.05$ were all significant. The pattern across these was clear: a larger average increase from binaural to dichotic conditions was observed in the speech task (5 to 12) than in the pitch task (7 to 8) and further, the increase was stronger in the $\mathrm{RH}$ (7 to 14) than the LH (5 to 7). Further, consistent with the first two interactions speech activated $\mathrm{RH}$ sites to a slightly larger extent $(\mathrm{RH}=12)$ than did pitch $(\mathrm{RH}=9)$ in this region (the relevant LH means were 5 for speech and 6 for pitch). Clearly then, the results from these analyses are consistent with the patterns evident in the composite image (see Fig. 8).

\section{DISCUSSION}

The results from this study indicated that when experimental conditions increased demands on selec- 
Pitch Binaural

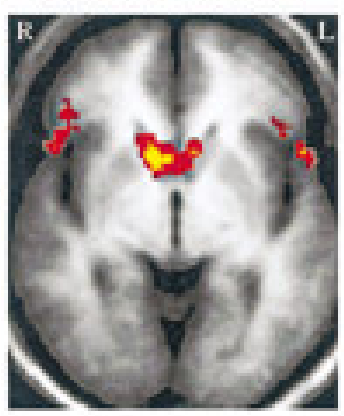

$z=1$

ection 2

$z=12$

ection 3 $z=24$
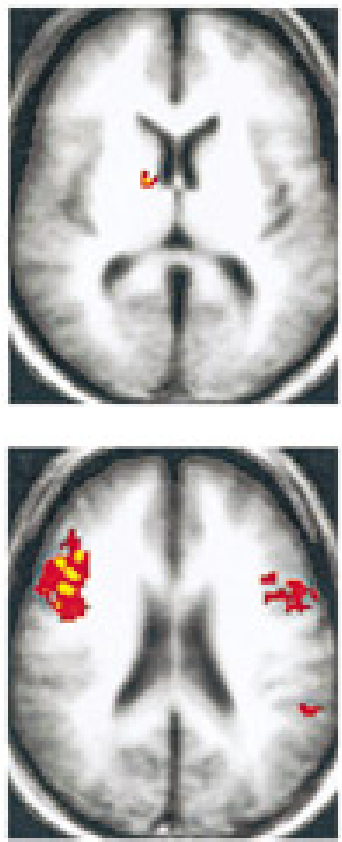

section 4 $z=36$

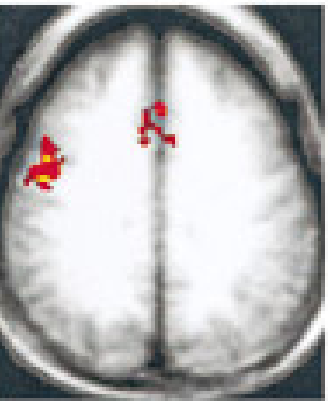

section 5 $z=48$

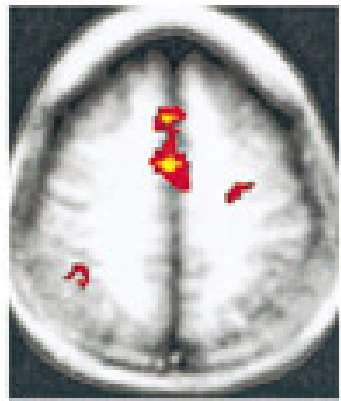

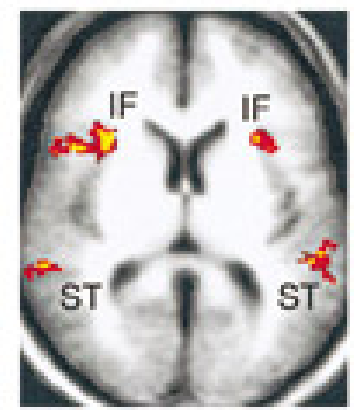
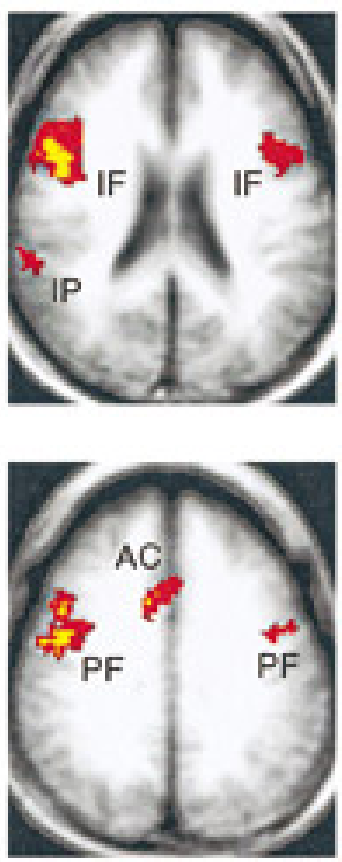

Pitch Dichotic
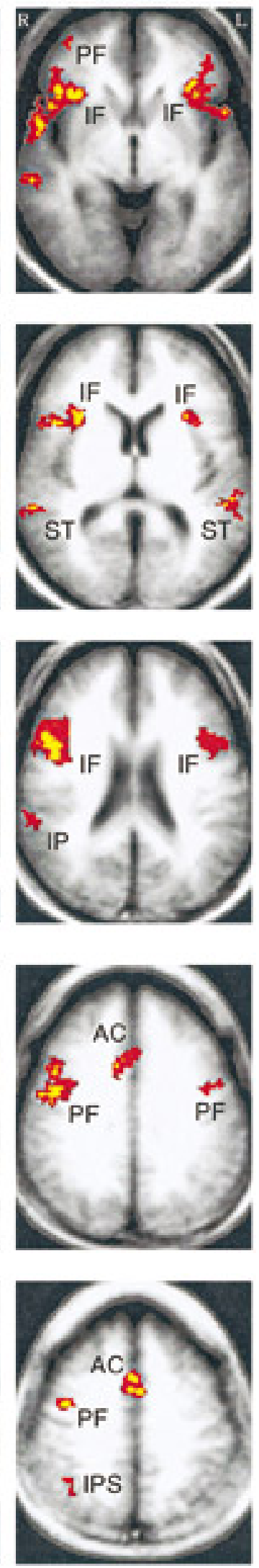
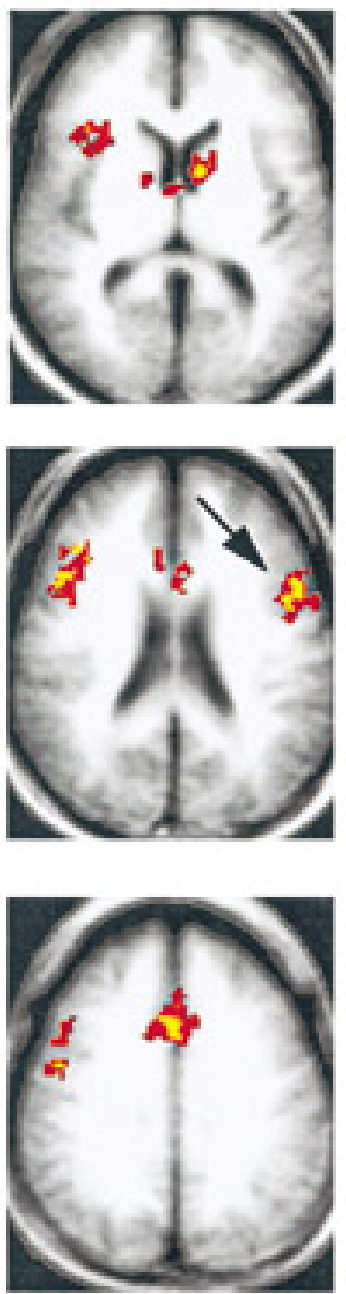

Speech Binaural

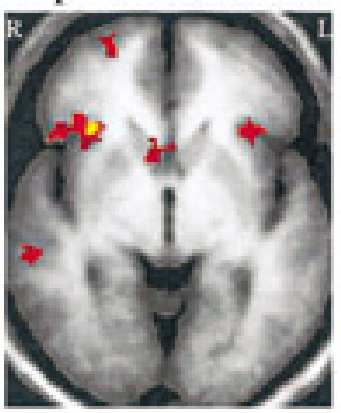

Speech Dichotic
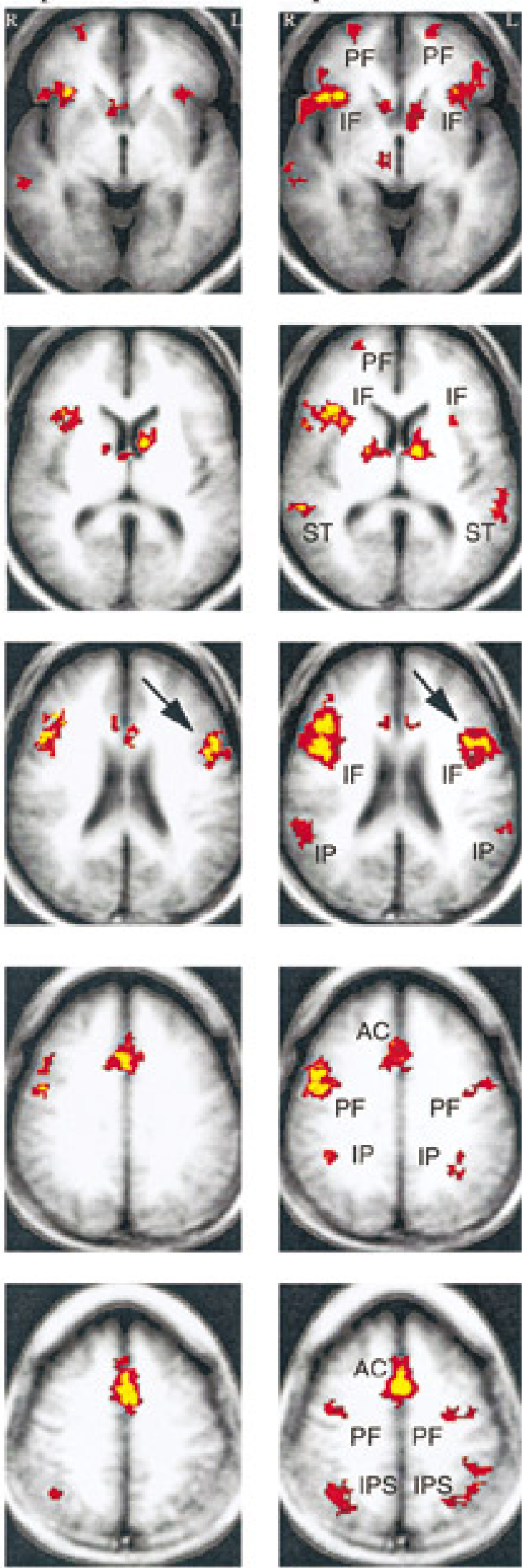
tive attention in the auditory modality, there was a systematic increase in activation within the parietal lobe. This effect was strongest in the inferior parietal lobule but evident, to a degree, in the superior parietal lobule and precuneous sites. Furthermore, this parietal increase was stronger in the $\mathrm{RH}$-though significantly so only in the precuneous. Also, the increased activation in the dichotic, relative to binaural, condition at these sites was not qualified by task; it was evident for both the speech and the pitch judgment tasks. By contrast, no increase (in fact, a nonsignificant decrease) in activation was observed in the anterior cingulate gyrus. This pattern of results confirmed, in general, the predictions made in theintroduction predicated on the cognitive/perceptual distinction (Posner and Dehaene, 1994).

Significant increases in activation in the dichotic conditions were also found at [B.A.] 22 in the superior temporal gyrus (bilaterally) and in [B.A.] 44 in the inferior frontal gyrus (primarily in the RH). These regions have been strongly implicated as basic sites relevant to spoken language processing (cf. Zatorre et al., 1992). Furthermore, a bilateral increase was observed in the prefrontal dorsolateral region ([B.A.] 46). Lesions to this region are known to be associated with difficulties in response inhibition in attention demanding tasks (Diamond et al., 1994). Thus it makes sense that, in the present experiment, the demands of inhibiting one channel and attending to another were associated with increased demands on this region. At the more anterior aspect of the prefrontal cortex ([B.A.] 10, 9 , and 8), a complex attention condition by task interaction was observed. At these sites, activation increased from binaural to dichotic conditions for the speech task, while a reliable decrease was observed for the pitch task. This complex interaction is not easily interpreted but was nevertheless quite robust. Further studies clearly will be needed to explore this region more analytically. A nearby site, [B.A.] 6, was shown to respond to the attentional variable, but only in the $\mathrm{RH}$ and primarily for the speech judgment task.

\section{Anterior vs Posterior Differences}

The results provided support for the hypothesis that the control of perception (manifest in selective attention tasks) is strongly associated with parietal function. Parietal sites responded strongly to the binaural/ dichotic manipulation. Further, the cingulate gyrus, thought to be most associated with control of decision and action, while strongly activated in both conditions (see Fig. 1), was uninfluenced by the sel ective attention manipulation. It seems reasonable to conclude that once the perceptual information had been processed in the more difficult dichotic conditions, decision and response execution was comparable with the binaural condition. The view that the cingulate region is associated with cognitive, but not perceptual, attentional control therefore would account for the failure to observe activation differences in the two attention conditions. As noted in the introduction, there is ample evidence that this ROI does respond to increased cognitive difficulty in divided attention tasks, Stroop tasks, and novel word generation tasks (Corbetta et al., 1991; Fiez and Petersen, 1993; Pardo et al., 1990; Raichle et al., 1994). Its failure to respond to the binaural/dichotic manipulation suggests that this region is not associated with control of selective attention. However, the anterior/posterior distinction appears too simplistic in general, in that several frontal lobe ROI s did respond to the sel ective attention manipulation. Increased activation at both temporal and frontal sites, coupled with the parietal increases, suggests that the physiological consequences of increased demands on auditory selective attention are broadly distributed throughout the cortex.

With respect to differences between speech and pitch judgments, previous neuroimaging studies (cf. Zatorre et al., 1992) have converged with numerous behavioral evidence suggesting an overall greater LH specialization for speech and language (cf. Luh and Levy, 1995; Studdert-Kennedy and Shankweiler, 1970). In the present investigation, we found an overall greater $\mathrm{RH}$ than $\mathrm{LH}$ activation across both tasks and both atten-

FIG. 8. Composite activation images for the 25 subjects (one image for each of the four subtraction conditions in each of five slices). Each anatomical region of interest with corresponding Brodmann's areas was specified by Talairach coordinates (see Methods and Table 1). Activated pixels with median $t$ values $>0.50$ (from the 25 initial t maps) are shown in color. By convention the LH is shown on the right side of each image. Increased spatial extent of activation in the two dichotic conditions relative to the two binaural conditions is seen in following areas: inferior frontal region (IF), Brodmann area [BA] 47 in section 1 and [BA] 44 and 45 in sections 2 and 3; posterior aspect of superior temporal region (ST), [BA] 22 in section 2; inferior aspect of inferior parietal region (IP), [BA] 40 in section 3-this probably also includes superior aspect of posterior superior temporal region, also [BA] 40; and the intraparietal sulcus (IPS) in section 5, which involves the superior-medial aspect of the inferior parietal region ([BA] 40) and inferior-lateral aspect of superior parietal region ([BA] 7). There is relative increase from speech binaural to speech dichotic conditions within both the inferior and superior prefrontal regions (PF), [BA] 10 in section 1 , [BA] 9 in section 4, and [BA] 6 in section 5. Part of the increased LH activation in both speech conditions relative to both pitch conditions is seen in superior aspect of Broca's area within the inferior frontal region (arrows, section 3). The dichotic and binaural conditions produce similar activation in the anterior cingulate region (AC), [BA] 24 and 32 in section 4 and [BA] 24 and 6 in section 5 . Key: $R$, right hemisphere; $L$, left hemisphere; IF, inferior frontal region; PF, prefrontal region; ST, superior temporal region; IP, inferior parietal region; AC, anterior cingulate region; and IPS, intraparietal sulcus bordering inferior parietal and superior parietal regions. 
tion conditions. This pattern $(\mathrm{RH}<\mathrm{LH})$ may have been a consequence of differences in the attentional demands of the experimental tasks relative to the noise burst detection control or may have been due to the more complex decisions they entailed. Nevertheless, several results suggested that the speech task was associated with relatively greater $\mathrm{LH}$ activation, while the pitch task was generally associated with relatively greater $\mathrm{RH}$ activation. This was most clearly seen in [B.A.] 44 in the inferior frontal gyrus, in both the ROI analysis and the composite images. Further, in the middle temporal gyrus ([B.A.] 21 and 39) activation increases from binaural to dichotic conditions showed a complex interaction with task and hemisphere. Relatively stronger increases (from binaural to dichotic) for speech were seen in the $\mathrm{LH}$, while relatively stronger increases for pitch were seen in the $\mathrm{RH}$. Thus, at two ROI s, a speech/LH and pitch/RH pattern was observed.

Finally, it should be noted that we found no indication in this study that any single ROI responded uniquely to only one or the other task. We do not claim that such intrahemispheric differences in speech and pitch localization do not exist. It is likely that the operations engaged by the pitch judgment task are overlapping to a degree with the operations engaged by the speech task, even if partially distinct, and this would make it difficult to isolate relatively fine-scaled intrahemispheric localization differences. The present study simply allowed the detection of a general hemispheric difference between these speech and pitch tasks. Further speech/nonspeech manipulations would be needed to inform this issue.

Encouraged by our previous finding (Shaywitz et al., 1995) of a sex by hemisphere interaction in a (visually presented) phonological task (rhyme judgment) at sites within the inferior frontal gyrus, we examined this issue in the present study. We anticipated that any relevant sex differences with respect to hemispheric distribution would be confined to the speech tasks which also tap basic phonological processing. As discussed above, the results showed a generally greater $\mathrm{RH}$ activation across all tasks and conditions in this study, which contrasts with the Shaywitz et al. reading study, where an overall LH advantage was obtained. Nonetheless, in the inferior frontal gyrus females displayed a proportionately greater $\mathrm{RH}$ involvement in the speech task processing than males, for whom a slight LH advantage was observed. This pattern, though shifted in overall direction toward the $\mathrm{RH}$, is consistent with the results from the reading experiment (Shaywitz et al., 1995), where males showed a strong LH greater than $\mathrm{RH}$ pattern and females showed equivalent $\mathrm{LH}$ and $\mathrm{RH}$ activation.

The present paradigm allows for a comparison of performance under conditions in which differential demands are placed on auditory selective attention while holding decision and response execution constant. F rom these data, we have obtained a preliminary characterization of some of the neural systems associated with auditory selective attention. The parietal lobe-particularly the inferior parietal lobule, but to some extent superior parietal sites as well-appears to be strongly associated with control of selective attention in audition. Moreover, activation patterns vary systematically with attentional difficulty at ROIs such as [B.A.] 22 and [B.A.] 44, sites known to be associated with basic spoken language perception. Finally, while several frontal regions were affected by the attentional manipulation, the absence of any effect of this variable in the anterior cingulate gyrus, a site strongly implicated in divided attention and Stroop tasks, reinforces the need to distinguish attention systems that control perceptual processing from systems associated with the control of cognitive operations.

\section{ACKNOWLEDGMENTS}

This research was supported by grants from the National Institute of Child Health and Human Development (PO1 HD-21888 and P50 HD-2580). The participation of Kenneth Pugh, Leonard Katz, and Donald Shankweiler was also supported by National Institute of Child Health and Human Development Grant HD-01994, and of Dani Byrd by NIH Grant DC-00016, to Haskins Laboratories. We thank Norman Kim, Carmel LaPore, Heddy Serifine, I gnatius Mattingly, Louis Goldstein, Pailing Hsiao, Einar Mend, and the staff at the Yale Center for the Study of Learning and Attention for their contributions to this project. We also thank Carol Fowler for valuable feedback on the manuscript.

\section{REFERENCES}

Allport, A. 1989. Visual attention. In Foundations of Cognitive Science (M. I. Posner, Ed.), pp. 631-682. MIT Press, Cambridge, $\mathrm{MA}$.

Andreasen, N. C., Rajaprabhakaran, R., Cizadio, T., Arnt, S., Swayze, V. W., Flashman, L., O'Leary, D. S., Ehrhart, J ., and Yuh, W. T. C. 1994. Automated atlas-based dissection of human brain from MR images. Society for Magnetic Resonance in Medicine Conference, San Francisco, CA.

Broadbent, D. E. 1958. Perception and Communication. Pergamon, London.

Corbetta, M., Miezin, F. M., Dobmeyer, S., Shulman, G. L., and Peterson, S. E. 1991. Selective and divided attention during visual discrimination of shape, color, and speed: Functional anatomy by positron emission tomography. J . Neurosci. 11:2383-2402.

Corbetta, M., Miezin, F. M., Shulman, G. L., and Petersen, S. E. 1993. A PET study of visuospatial attention. J . Neurosci. 13:1202-1226.

Goldman-Rakic, P. S. 1987. Circuitry of primate prefrontal cortex and regulation of behavior by representational knowledge. In The Handbook of Physiology, Sec. 1, TheNervous System, Vol. V, Higher Functions of the Brain (F. Plum, and V. Mountcastle, Eds.), pp. 373-417. Am. Physiol. Soc., Bethesda, MD.

Hari, R., Hamalianen, M., Kaukoranta, E., Makela, S.-L., and Tiihonen, J. 1989. Selective listening modifies activity of the human auditory cortex. Exp. Brain Res. 74:463-470. 
Heilman, K. M., Bowers, D., Coslett, H. B., Whelan, H., and Watson, R. T. 1985. Directional hypokinesia: Prolonged reaction times for leftward movements in patients with right hemisphere lesions and neglect. Neurology 35:855-859.

Kahneman, D. 1973. Attention and Effort. Prentice Hall, Englewood Cliffs, NJ .

Luh, K. E., and Levy, J . 1995. Interhemispheric cooperation: Left is left and right is right, but sometimes the twain shall meet. J . Exp. Psychol. Hum. Percept. Perform. 21:1243-1258.

Navon, D. 1984. Resources-A theoretical soupstone? Psychol. Rev. 91:216-234.

Navon, D., and Gopher, D. 1979. On the economy of the human processing system. Psychol. Rev. 86:214-255.

Pardo, J . V., Pardo, P. J ., J aner, K. W., and Raichle, M. E. 1990. The anterior cingular cortex modulates mediates processing selection in the Stroop attentional conflict paradigm. Proc. Natl. Acad. Sci. USA 87:256-259.

Pardo, J. V., Fox, P. T., and Raichle, M. E. 1991. Localization of a human system for sustained attention by positron emission tomography. Nature 349:61-64.

Petersen, S. E., and Fiez, J . A. 1993. The processing of single words studied with positron emission tomography. Ann. Rev. Neurosci. 16:509-530.

Petrides, M., Alivisatos, B., Meyer, E., and Evans, A. C. 1993. Functional activation of the human frontal cortext during the performance of verbal working memory tasks. Neurobiology 90:878-882.

Posner, M. I. 1991. Editor's note: Attention as a cognitive neurosystem. J . Cogn. Neurosci. 3:303.

Posner, M. I., and Boies, S. J . 1971. Components of attention. Psychol. Rev. 78:391-408.
Posner, M. I., and Dehaene, S. 1994. Attentional networks. Trends Neurosci. 17:75-79.

Posner, M. I., and Petersen, S. E. (1990). The attention system of the human brain. Annu. Rev. Neurosci. 13:25-42.

Posner, M. I., and Presti, Presti, D. 1987. Selective attention and cognitive control. Trends Neurosci. 10:12-17.

Raichle, M. E., Fiez, J . A., Videen, T. O., MacLeod, M. K., Pardo, J . V., Fox, P. T., and Petersen, S. E. 1994. Practice related changes in human brain functional anatomy during nonmotor learning. Cereb. Cortex 4:8-26.

Roland, P. 1994. Brain Activation. Wiley, New York.

Schneider, W. and Shiffrin, R. M. 1977. Controlled and automatic human information processing. Psychol. Rev. 84:1-66.

Shaywitz, B. A., Shaywitz, S. E., Pugh, K. R., Constable, R. T., Skudlarski, P., Fulbright, R. K., Bronen, R. A., Fletcher, J . M., Shankweiler, D. P., Katz, L., and Gore, J . C. 1995. Sex Differences in the Functional Organization of the Brain for Language. Nature 373:607-609.

Studdert-Kennedy, M., and Shankweiler, D. 1970. Hemispheric specialization for speech perception. J . Acoust. Soc. Am. 48:579-594.

Treisman, A. M. 1964. Monitoring and storage of irrel evant messages in selective attention. J . Verb Learn. Verb Beh. 3:449-459.

Wickens, C. D. 1980. The structure of attentional resources. In Attention and Performance (R. S. Nickerson, Ed.). Vol. VIII. Erlbaum, Hillsdale, NJ .

Woldorff, M. G., Gallen, C. G., Hampson, S. A., Hillyard, S. A., Pantev, C., Sobel, D., and Bloom, F. E. 1993. Neurobiology 90:8722-8726.

Zatorre, R. J ., Evans, A. C., Meyer, E., Gjedde, A. 1992. Lateralization of phonetic and pitch discrimination in speech processing. Science 256:846-849. 